\title{
Efficacy of gluten-free diet in patients with rheumatoid arthritis
}

\author{
V. Bruzzese ${ }^{1}$, P. Scolieri', J. Pepe ${ }^{2}$ \\ 'U.O.C. Internal Medicine and Rheumatology, ASL Roma 1, \\ Hospital S. Spirito-Nuovo Regina Margherita, Rome, Italy; '2Department of Clinical, Internal, \\ Anesthesiology and Cardiovascular Sciences, La Sapienza University, Rome, Italy
}

\begin{abstract}
Recent research has increasingly shown that depending on the foods we eat, gut flora may be affected by an inflammatory or anti-inflammatory response, thus playing an important role in inflammatory autoimmune diseases, such as rheumatoid arthritis or gastroenterological disorders. Gluten seems to be a glycoprotein with a clinically relevant inflammatory effect. Several observational studies and anecdotal cases reported a correlation between gluten and various diseases, including autoimmune diseases, such as rheumatoid arthritis. This study aimed to evaluate whether gluten-free diet could be effective in controlling inflammation and ongoing rheumatoid arthritis symptoms.

We report 4 cases of patients with long-standing rheumatoid arthritis with no response to several conventional and biotechnological drugs, treated with a gluten-free diet concurrently with the drug therapy. Our patients presented different degrees of response to the diet, in terms of disease remission and improvement of symptoms. Our cases confirm that a gluten-free diet may improve symptoms of rheumatoid arthritis, even in patients resistant to conventional drug therapies.
\end{abstract}

Key words: Gluten-free diet, rheumatoid arthritis, celiac disease, non-celiac gluten sensitivity.

Reumatismo, 2020; 72 (4): 213-217

\section{INTRODUCTION}

$\mathrm{R}$ heumatoid arthritis (RA) is a chronic inflammatory disease, of unknown etiology, in which genetic predisposition and various environmental factors play a key role in the development of autoimmunity and inflammation.

Beside the best known environmental factors, such as smoking, bacterial/viral infections and stress, recent studies have also focused on the possibility that food may cause systemic inflammation. A series of observations suggest that gut flora may play an important role in the development of chronic inflammatory diseases, both in gastroenterological and rheumatological settings. Depending on the foods, the gut microbiome may exert an inflammatory or anti-inflammatory response.

Gluten is a glycoprotein present in some cereals. Celiac disease (CD) is the most severe form of gluten intolerance. A milder form is the non-celiac gluten sensitivity (NCGS). The latter, like $\mathrm{CD}$, has been associated not only with irritable bowel syndrome but also with various other diseases, including RA (1). Gluten may be also a triggering factor in various clinical conditions, such as ataxia, multiple sclerosis, schizophrenia, and fibromyalgia, which have been all associated with gluten consumption, even in the absence of an established CD or NCGS (2). Alongside conventional drug therapy, in many chronic inflammatory diseases, including RA, there is additional space for complementary therapy, especially nutritional therapy. In this context a gluten-free diet (GFD) has been shown to be a possible treatment for RA and psoriatic arthritis $(3,4)$.

In light of the above clinical observations, we added a GFD in 4 patients suffering from long-standing RA without a sufficient improvement with biological therapy.

\section{Case n. 1}

A 44-year-old man had been affected by seronegative RA with polyarticular involvement since the age of 30 . At the age of 32 , he started a pharmacological treatment with
Corresponding author: Palma Scolieri Medicina Interna e Reumatologia Ospedale Nuovo Regina Margherita Via E. Morosini 30 - 00153 Roma, Italy E-mail: palma.scolieri@gmail.com 
methotrexate (MTX), which was discontinued after a few months due to liver damage. In the following years, several treatments were attempted with various biological drugs (etanercept, adalimumab, infliximab and tocilizumab), but all were discontinued due to ineffectiveness.

At the age of 39 , he began a therapy with abatacept $(750 \mathrm{mg} / \mathrm{month})$, resulting in a good disease control. After two years of therapy, the patient independently decided to discontinue abatacept. For approximately one year, the patient did not follow any therapy except for non-steroidal anti-inflammatory drugs (NSAIDs).

In February 2015, the patient presented with a worsened disease activity (DAS 28 CRP $=5.8$ ) with important functional impotence. $\mathrm{He}$ restarted therapy with abatacept and achieved an improvement after approximately one year (DAS 28=3.5) but did not reach any minimal disease activity or remission, thus remaining on daily NSAIDs.

A GFD was then suggested, despite immunological tests (antigliadin antibodies, anti-endomysium and anti-transglutaminase antibodies) for $\mathrm{CD}$ were negative. The patient did not have any abdominal symptoms suggestive of CD or NCGS.

After one month of GFD, the patient showed a progressive improvement and, after 5 months, he achieved full remission (DAS $28<2.6$ ). After 10 months of GFD, the state of remission and general well-being persisted and the patient decided to suspend abatacept against the advice of his rheumatologist, and continuing only GFD. After 2 months of therapy discontinuation, his conditions worsened (DAS 28=5.2) with functional impotence. Therefore, abatacept was started again and the GFD instituted again.

\section{Case n. 2}

A 65-year-old woman had been affected by seropositive RA since the age of 53, initially treated with MTX (15 mg/week) and glucocorticoids (GC) without benefit. One year after the diagnosis, she was treated with etanercept $(50 \mathrm{mg} /$ week $)$ associated with MTX, and obtained a good disease control (DAS 28=3.1). For about 7 years, her conditions remained stationary but then worsened
(DAS 28=6.1). Etanercept was discontinued to start abatacept (750 mg/month), with partial control of her disease. After two years, the patient presented worsening joint pain and functional impotence (DAS 28=5.4). Abatacept was then discontinued and tocilizumab (162 mg/week) plus MTX was started. After five months of therapy, the disease was still poorly controlled (DAS $28=4.8$ ).

After testing for $\mathrm{CD}$ with negative results, and without abdominal symptoms suggesting NCGS, GFD associated with the ongoing drug therapy was recommended. After one month of GFD, the patient achieved remission (DAS $28<2.6$ ), with complete functional recovery. The patient decided to stop the diet after about 3 months, while continuing the same drug therapy. Within 3-4 months, joint pain progressively recurred and her clinical conditions returned to the pre-diet state.

\section{Case n. 3}

A 54-year-old woman had been affected by seropositive RA since the age of 42 . She was treated sequentially with MTX, leflunomide, sulfasalazine and GC, without success. In December 2012, a high disease activity (DAS 28=5.2) was seen. The patient began golimumab (50 mg month) in mono-therapy because of gastrointestinal intolerance to MTX. Good disease control was achieved for four years. In May 2017, the patient experienced a disease flare (DAS 28=3.8). She was tested for $C D$, with negative results. Even though abdominal symptoms were absent, we recommended a GFD in adjunction to her pharmacological therapy. In the first month, a significant improvement in joint symptoms was obtained (DAS 28=2.8). Subsequently, the patient suspended the GFD, for she felt joint pain was worsening. After that, the disease progressively worsened with significant functional impotence. It was therefore decided to stop golimumab and prescribe tocilizumab (162 mg/week), without GFD. After 4 months, a persistent remission was obtained (DAS 28<2.6).

\section{Case n. 4}

A 43-year-old woman had been affected by seropositive RA since the age of 23 . 
She was initially treated with gold salts for many years, with good success. In 2010, due to a severe exacerbation of the disease (DAS 28=5.6), we started biological treatment (first etanercept and later abatacept) in monotherapy because of MTX intolerance; both were discontinued for ineffectiveness. Thereafter, treatment with adalimumab (40 mg twice/monthly) and GC (methylprednisolone $8 \mathrm{mg} /$ day) was administered, obtaining a good disease control (DAS 28=3.2), without ever achieving remission and without ever suspending GC. After about five years of this therapy, the patient experienced progressive pain in the wrists and fingers, with swelling and severe functional impairment, especially of the right wrist (DAS 28=5.2).

After testing for $\mathrm{CD}$, with negative results, and even in absence of abdominal symptoms, we recommended a GFD, in association with the same drug therapy. After 10 days from the beginning of the diet, the patient experienced progressive improvement, with subsequent disappearance of pain and swelling in the fingers and important improvement in the wrists, with almost total functional recovery (DAS 28=3.0), permitting to taper the dose of GC.

\section{SUMMARY OF DATA}

Considering the overall population examined, the main data of the patients before and after GDF are reported in Table I. A statistically significant decrease was observed in the DAS 28 -CRP $(5.05 \pm 0.86 v s$
Table I - Main parameters analyzed before and after GFD. Data are reported as mean \pm one standard deviation.

\begin{tabular}{|l|c|c|}
\hline & Basal & After GFD \\
\hline ESR & $8 \pm 5.35$ & $6.5 \pm 5.92$ \\
\hline CRP & $1.43 \pm 2.38$ & $0.2 \pm 0.1$ \\
\hline DAS28-CRP & $5.05 \pm 0.86$ & $2.63 \pm 0.36^{*}$ \\
\hline TJ & $14 \pm 4.32$ & $3.5 \pm 4.12^{*}$ \\
\hline SJ & $4.5 \pm 1.73$ & $0 \pm 0^{*}$ \\
\hline VAS & $5.25 \pm 1.89$ & $1.5 \pm 1.29^{*}$ \\
\hline
\end{tabular}

${ }^{*} p<0.05$. ESR, erythrocyte sedimentation rate; CRP, C-reactive protein; DAS28-CRP, Disease Activity Score; TJC, tender joint count; SJC, Swollen Joint Count; VAS, Visual analogue scale.

$2.63 \pm 0.36, \mathrm{p}<0.05)$, the Tender Joint Count (TJC) $(14 \pm 4.32$ vs $3.5 \pm 4.12, \quad \mathrm{p}<0.05)$, Swollen Joint Count (SJC) $(4.5 \pm 1.73$ vs $0 \pm 0, \mathrm{p}<0.05)$, and the VAS $(5.25 \pm 1.89$ vs $1.5 \pm 1.29, \mathrm{p}<0.05)$. Figure 1 shows the value of the DAS 28-CRP before and after GDF for each patient.

\section{DISCUSSION AND CONCLUSIONS}

Nutrition and gut microbiota could play a role as a triggering factor for systemic inflammation and some rheumatic diseases, such as RA; such a role remains controversial, however. Our 4 clinical cases cannot be final and conclusive, but they can contribute to partially explain the possible clinical outcomes after starting a GFD in RA patients. First, all four patients were multi-resistant to many conventional and biotechnologi-

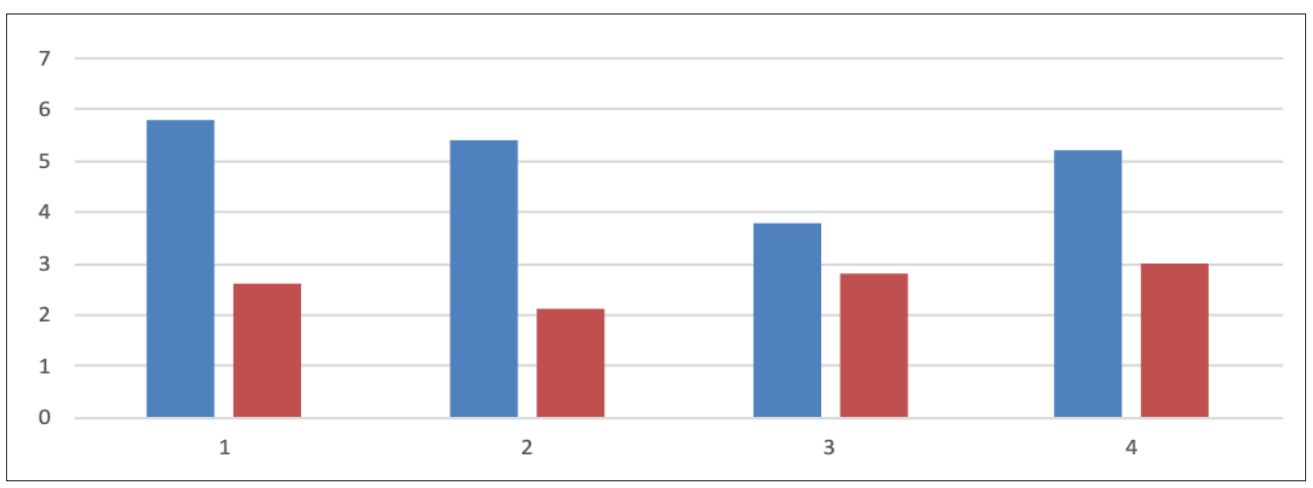

Figure 1 - DAS 28-PCR in the 4 patients examined. The columns in blue represent the basal value, those in orange the value after the GFD. 
cal drugs. These patients, while undergoing therapy with biotechnological drugs, started a GFD during a period of severe exacerbation of their disease. Over time, they changed several therapies but experienced an improvement only with the GFD. The placebo effect therefore does not appear to be very likely in patients already heavily treated before GFD, although it cannot be ruled out.

Second, most studies consider patients that are not treated with drug therapy, but only on a GFD for the treatment of RA or other autoimmune inflammatory disorders. Therefore, our cases are the first in which a GFD is associated with a conventional and biotechnological drug therapy, which is currently the most effective therapy for this disease. Thus, according to our experience, GFD is not contraindicated when a previous treatment has been started, and a synergistic effect cannot be excluded.

Third, in our first case, when the biotechnological drug had been discontinued upon the patient's autonomous decision, there was disease worsening, despite the GFD. Therefore, it seems unlikely that disease improvement could be linked to a diet placebo effect. However, this particular case shows that GFD cannot be considered a sufficient therapy for autoimmune inflammatory diseases but only a complementary support, more or less important, to the success of pharmacological therapies that did not have or have lost their effectiveness.

The issue of the importance of nutrition on systemic autoimmune diseases is still far from finding adequate answers. Undeniably, certain foods may affect gut microbiota with a pro-inflammatory response (7). Having said that, how much a diet without pro-inflammatory foods can actually influence the course of an autoimmune disease, such as RA, is far from being clarified. Moreover, it is likely that in some patients with intestinal mucosa damage and gut leakage, possibly caused by pharmacological therapies, certain foods, like gluten, may trigger arthritis. It also seems confirmed that intestinal dysbiosis, with the presence of the Prevotella species, may trigger RA through a mechanism of molecular mimicry between the $\mathrm{T}$ cells of the microorganism and the epitopes of the host (8). In a state of intestinal dysbiosis, gluten may have a greater inflammatory effect than on a subject without dysbiosis.

Damage to the intestinal barrier, resulting in increased permeability to both bacterial and food products, has also been found in patients with Crohn's disease. In these patients a high value of zonulin ( $\mathrm{ZO}$ ), a biomarker of intestinal permeability, has been reported. Elevated values of $\mathrm{ZO}$ are accompanied by increased inflammatory damage and cytokines (9). Gliadin has also been shown to induce an increase in intestinal permeability in healthy subjects, through an increase in the concentration of ZO (10).

In patients with RA, high anti-gliadin antibody levels were detected in the intestinal fluid compared to healthy controls, thus supporting a probable antigenic action of gluten (11). Anti-gliadin and anti-tissue transglutaminase antibodies were also detected in other inflammatory autoimmune disorders such as multiple sclerosis (12).

The inflammatory effect of gluten also develops through various other mechanisms. Gluten activates Toll-like receptor 4 and stimulates the production of various proinflammatory cytokines, such as IL-6, IL-1 and especially IL-17. It also determines a dysbiosis through a modification of the microbiota in a proinflammatory sense and can alter the epigenetic program, resulting in a lack of methylation of genes resulting in higher incidence of disease (13).

Our 4 cases seem to suggest that a correlation of the inflammatory state with gluten intake can be plausible, confirming the scientific trend of the last years (14). However, it remains uncertain whether and to what extent RA patients may benefit from a GFD. Considerations and questions that would need randomized controlled trials.

In conclusion, our observations indicate that in patients with RA, multi-resistant to both conventional and biotechnological therapies, an attempt with a GFD can be made and can produce good therapeutic results. An aspect that must however be taken into consideration, and which is the subject of many studies, concerns the possibility that GFD may increase cardio-vascular risk fac- 
tors (CV). This would not be desirable in a disease such as RA, in which CV risk is intrinsically increased. While some studies report an increased risk of hepatic steatosis and metabolic syndrome during GFD (15), other meta-analysis studies deny an increased $\mathrm{CV}$ risk in patients following longterm GFD (16). This issue remains controversial, but the more powerful studies seem to confirm an absolute indifference of gluten on CV risk factors (17) or even a protective effect of GFD on the components of the metabolic syndrome (18).

In conclusion, our observations indicate that in patients with RA, multi-resistant to both conventional and biotechnological therapies, an attempt with a GFD can be made and can give good therapeutic results.

However, the question remains which RA patients can benefit from a GFD: a consideration that deserves double-blind controlled studies for an answer.

\section{Conflicts of Interest}

The authors have no conflicts of interest to declare.

\section{Acknowledgements}

We thank prof. Yehuda Shoenfeld for his helpful comments.

\section{Key points}

The role of gluten in triggering rheumatic diseases has been suggested, but the topic is still debated.

Our data suggest that gluten-free diet may be useful in reducing symptoms in rheumatoid arthritis patients.

\section{REFERENCES}

1. Volta U, Bardella MT, Calabrò A, et al. An Italian prospective multicenter survey on patients suspected of having non-celiac gluten sensitivity. BMC Medicine. 2014; 12: 85.

2. San Mauro Martin I, Garicano Vilar E, Collado Yurrutia L, et al. Is gluten the great etiopathogenetic agent of disease in the XXI century? Nutr Hosp. 2014; 30: 1203-10.

3. El-Chammas K, Danner E. Gluten-free diet in nonceliac disease. Nutr Clin Pract. 2011; 26: 294-9.

4. D'Erme AM, Kovacikova Curkova A, Agnoletti AF, et al. Gluten-free diet as a therapeutic approach in psoriatic patients: if yes, when. $G$
Ital Dermatol Venereol. 2015; 150: 317-20.

5. Hafstrom I, Ringertz B, Spangberg A, et al. A vegan diet free of gluten improves the signs and symptoms of rheumatoid arthritis: the effects on arthritis correlate with a reduction in antibodies to food antigens. Rheumatology (Oxford). 2001; 40: 1175-9.

6. Elkan AC, Sjoberg B, Kolsrud B, et al. Glutenfree vegan diet induces decreased LDL and oxidized LDL levels and raised atheroprotective natural antibodies against phosphorycholine in patients with rheumatoid arthritis: a randomized study. Arthritis Res Ther. 2008; 10: R34.

7. Cutolo M, Nikiphorou E. Don't neglet nutrition in rheumatoid arthritis! RMD Open. 2018; 4: e000591.

8. Pianta A, Arvikar SL, Strle K, et al. Two rheumatoid arthritis-specific autoantigens correlate microbial immunity with autoimmune responses in joints. J Clin Invest. 2017; 127: 2946-56.

9. Vanuytsel T, Vermeire S, Cleynen I. Thr role of Haptoglobin and its related protein, zonulin, in inflammatory bowel disease. Tissue Barriers. 2013: 27321.

10. Drago S, EJ Asmar R, Di Pierro M, et al. Gliadin, zonulin and gut permeability: effect in celiac and non-celiac intestinal mucosa and intestinal cell lines. Scan J Gastroenterol. 2006; 41: 408-19.

11. Hvatum M, Kanerud L, Hallgren R, et al. The gut-joint axis: cross reactive food antibodies in rheumatoid arthritis. Gut. 2006; 55: 1240-7.

12. Shor DB, Barzilai O, Ram M, et al. Gluten sensitivity in multiple sclerosis: experimental myth or clinical truth? Ann N Y Acad Sci. 2009; 1173: 343-9.

13. Lerner A, Shoenfeld Y, Matthias T. Adverse effects of gluten ingestion and advantages of gluten withdrawal in nonceliac autoimmune disease. Nutr Rev. 2017; 75: 1046-58.

14. Lerner A, Mathias T. Rheumatoid arthritis-celiac disease relationship: joints get that gut feeling. Autoimmun Rev. 2015; 14: 1038-47.

15. Ciccone A, Gabrieli D, Cardinale R, et al. Metabolic alteration in celiac disease occurring after following a gluten free diet. Digestion. 2019; 100: 262-8.

16. Potter MD, Brienesse SC, Walker NM, et al. Effect of gluten- free diet on cardiovascular risk factors in patients with coeliac disease: a systematic review. J Gastroenterol Hepatol. 2018; 33: 781-91.

17. Lebwohl B, Cao Y, Chan AT. Long term gluten consupition in adult without celiac disease and risk of coronary heart disease: prospective cohort study. BMJ. 2017; 357: J1892.

18. Ehtcshami M, Shaker Hosseini R, Scdaghat F, et al. The effect of gluten free diet on components of metabolic syndrome: a randomized clinica trial. Asian Pac J Cancer Prev. 2018; 19: 2979-84. 